Klinikdirektor an der Neurologischen Klinik der Charité, von 2004 bis 2007 Klinikdirektor am Campus Benjamin Franklin der Charité. Seit 2007 ist er Direktor am MPI für Kognitions- und Neurowissenschaften sowie Klinikdirektor der Klinik für Kognitive Neurologie am Uniklinikum Leipzig. Prof. Villringer leitet seit 1999 das bundesweite Kompetenznetz Schlaganfall und hat 2006 im Rahmen der Exzellenzinitiative die Berlin School of Mind and Brain gegründet, deren Sprecher er seither ist. Sein Forschungsschwerpunkt sind der Schlaganfall und seine Risikofaktoren.

Annette Horstmann (geb. 1977 in Hamburg), studierte Biologie an der RuhrUniversität in Bochum. Nach einem einjährigen Forschungsaufenthalt am Max-Planck-Institut für Kognitions- und Neurowissenschaften in Leipzig (Arbeitsgruppe „Attention \& Awareness“, Prof. John-Dylan Haynes) und der Promotion im Rahmen der „Internationalen Graduiertenschule Biowissenschaften“ in Bochum war sie von 2008 bis 2010 als PostDoc in der Abteilung für Neurologie desselben Institutes tätig. Von 2010 bis 2013 arbeitete sie als wissenschaftliche Mitarbeiterin in einem Kooperationsprojekt zwischen dem MPI und dem IFB Adipositas-Erkrankungen, Universitätsmedizin Leipzig. Dr. Horstmann leitet seit 2013 die eigenständige Nachwuchsforschungsgruppe „Entscheidungsverhalten bei Adipositas: Neurobiologie, Verhalten und Plastizität" im Rahmen des IFB Adipositas-Erkrankungen in Kooperation mit dem MPI für Kognitions- und Neurowissenschaften. Ihr Forschungsschwerpunkt sind die neurobiologischen Grundlagen der Adipositas beim Menschen.

\section{Korrespondenzadressen}

\section{Dr. Annette Horstmann}

Max-Planck-Institut für Kognitions-

und Neurowissenschaften

Kognitive Neurologie

Stephanstr. $1 \mathrm{~A}$

04103 Leipzig

Tel.: $\quad+4934199402258$

E-Mail: horstmann@cbs.mpg.de

\author{
Prof. Dr. Arno Villringer \\ Max-Planck-Institut für Kognitions- \\ und Neurowissenschaften \\ Kognitive Neurologie \\ Stephanstr. $1 \mathrm{~A}$ \\ 04103 Leipzig \\ Tel.: $\quad+4934199402220$ \\ E-Mail: villringer@cbs.mpg.de
}

\title{
Lieferung auf Abruf: Exosomen als "Care“-Pakete von Gliazellen für gestresste Neurone
}

\author{
Eva-Maria Krämer-Albers und Carsten Frühbeis
}

Zusammenfassung

Die Kommunikation zwischen Zellen ist eine grundlegende Voraussetzung für reibungslose Abläufe im Nervensystem. Gliazellen besitzen dabei eine Vielzahl von Aufgaben, die in enger Abstimmung mit Neuronen wahrgenommen werden. Forschungen der letzten Jahre zeigen, dass Zellkommunikation auch über den Austausch von extrazellulären Vesikeln stattfindet, die ebenfalls von Gliazellen und Neuronen sezerniert werden. Zu den extrazellulären Vesikeln gehören Exosomen und Mikrovesikel, welche Proteine und Ribonukleinsäuren zu Zielzellen transportieren. Nach erfolgtem Transfer können diese Komponenten dann den Phänotyp der Zielzelle verändern. In diesem Artikel diskutieren wir Eigenschaften und Funktionen von extrazellulären Vesikeln im Allgemeinen und speziell im zentralen Nervensystem. Dort geben myelinisierende Oligodendrozyten in Antwort auf Neurotransmittersignale Exosomen ab, die von Neuronen aufgenommen werden und neuroprotektive Eigenschaften besitzen.

\section{Abstract}

Delivery on call: Exosomes as „Care packages“ from glia cells for stressed neurons. Communication between cells is a basic requirement for proper nervous system function. Glia cells execute versatile functions, which operate in close coordination with neurons. Recent research revealed that cell communication is mediated by the exchange of extracellular vesicles, which are also secreted by glia cells and neurons. Extracellular vesicles comprise exosomes and microvesicles, which deliver proteins and ribonucleic acids to target cells. As a result of transfer, the vesicle cargo components can modulate the phenotype of recipient cells. Here, we discuss the characteristics and functions of extracellular vesicles in general and in particular in the central nervous system. There, myelinating oligodendrocytes release exosomes in response to neurotransmitter signals, which are internalized by neurons and exhibit neuroprotective functions.

Keywords: oligodendrocytes; extracellular vesicles; exosomes; cell-cell communication; axon-glia interaction

\section{Einleitung}

Gliazellen partizipieren aktiv an den verschiedensten Prozessen im Nervensystem. Die Vorstellung vom ursprünglich durch Rudolf Virchow terminierten „Leim“ des Nervengewebes ist längst überholt und die Liste der Fähigkeiten von Gliazellen erweitert sich kontinuierlich. Mikroglia, Astrozyten und Oligodendrozyten sind sowohl für die Entwicklung als auch für die alltäglichen Funktionen des Gehirns von enormer Bedeutung: Sie steuern das Wachstum von Axonen, gewährleisten die Nährstoffversorgung von Neuronen, modulieren Signalverarbeitungsprozesse, sorgen für elektrische Isolation (Myelinbil- dung) und kontrollieren Immunprozesse. Die Koordination dieser Abläufe hängt in besonderem Maße von der Kommunikation zwischen Gliazellen und Neuronen ab. Nach der klassischen Sicht findet Zellkommunikation entweder durch direkten Zellkontakt oder die parakrine Wirkung löslicher Mediatoren statt. Allerdings hat man im Laufe des letzten Jahrzehnts festgestellt, dass auch ganze Vesikel zwischen Zellen ausgetauscht werden, die eine Kollektion von Biomolekülen, gleichsam als Paket, zu Zielzellen transportieren können. Die ,angelieferten“ Moleküle können dann direkt in den Zielzellen agieren (Thery 2011). Aktuelle Forschungen zeigen, dass auch die Zellen des Nervensystems Vesikel 


\section{Exkurs}

\section{Extrazelluläre Vesikel und ihre Herkunft}

Es ist schon lange bekannt, dass sich im Laufe der Apoptose große Vesikel von sterbenden Zellen abschnüren. Sogenannte apoptotische Körper enthalten DNA-Fragmente, RNA-Moleküle und Überreste von Mitochondrien. Gesunde Zellen hingegen sezernieren hauptsächlich zwei Typen von Vesikeln: Exosomen und Mikrovesikel. Letztere schnüren sich direkt von der Plasmamembran $\mathrm{ab}$ und weisen eine heterogene Größenverteilung auf (bis zu $1000 \mathrm{~nm}$ im Durchmesser). Exosomen dagegen stammen aus dem endosomalen System und sind kleiner (50-100 nm im Durchmesser). Sie entsprechen den intralumenalen Vesikeln (ILVs) der Multivesikulären Endosomen (MVEs), die durch Fusion mit der Plasmamembran ins extrazelluläre Milieu freigesetzt werden. Die Exosomenbiogenese setzt daher die Bildung der MVEs voraus, die durch das nach innen gerichtete Einstülpen der endosomalen Membran und Abschnüren der ILVs entstehen. Die MVEs können entweder mit Lysosmen fusionieren, was zum Abbau der ILVs und ihrem Inhalt führt, oder mit der Plasmamembran fusionieren, um Exosomen freizusetzen. Die Proteinsortierung in Exosomen läuft an der endosomalen Membran ab, wozu der ESCRT (,Endosomal Sorting Complex Required for Transport") -Komplex benötigt wird. Des Weiteren existieren ESCRT-unabhängige Mechanismen. Das Enzym Sphingomyelinase kann die lokale Bildung von Ceramid katalysieren, was eine Abschnürung der ILVs begünstig. Außerdem kann eine Klasse von Transmembranproteinen, die sogenannten Tetraspanine, beteiligt sein. Das Andocken der MVEs an der Plasmamembran ist Voraussetzung für die darauf folgende Fusion und Freisetzung der Exosomen und wird von kleinen GTPasen der Rab-Familie (Rab11, Rab27 und Rab35) kontrolliert. Exosomen enthalten charakteristische Lipide, verschiedene RNA-Species (hauptsächlich mRNA und miRNA), an der Exosomenbiogenese beteiligte Proteine, Tetraspanine, Integrine und Hitzeschockproteine. Außerdem tragen sie zelltypspezifische Komponenten, die sowohl die Identität als auch den Status der Donorzelle widerspie- geln. Auf der anderen Seite enthalten sie keine ER- oder mitochondrialen Proteine. Über die spezifische Zusammensetzung von Mikrovesikeln und deren Biogenese ist weniger bekannt. Allerdings werden für die Bildung der Mikrovesikel auch Faktoren eingesetzt, die bei der Exosomenbiogenese eine Rolle spielen.

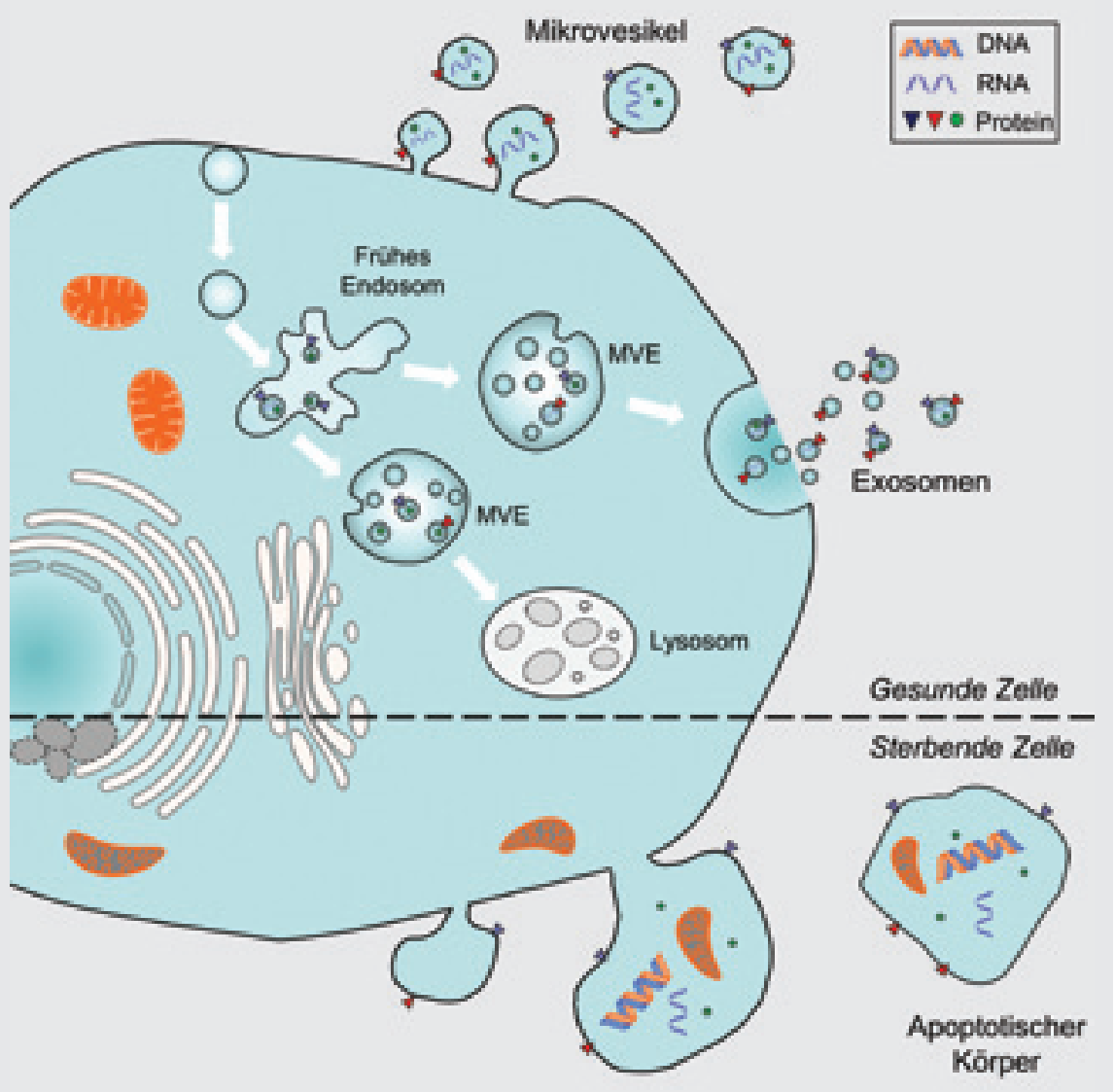

Abb. Exkurs: Freisetzung von Exosomen und Mikrovesikeln. Mikrovesikel schnüren sich direkt von der Plasmamembran ab, während Exosomen aus dem endosomalen System stammen und durch die Fusion der MVEs mit der Plasmamembran freigesetzt werden. Andere MVEs fusionieren mit Lysosomen. Sowohl Exosomen als auch Mikrovesikel tragen Proteine und RNA. Sterbende Zellen geben apoptotische Körper ab, die DNA, RNA und Reste von Mitochondrien enthalten.

als Fähren für Biomoleküle zur interzellulären Kommunikation benutzen (Frühbeis et al. 2013a).

Die von Zellen abgegebenen Vesikel werden allgemein als extrazelluläre Vesikel bezeichnet und lassen sich nach Herkunft, Zusammensetzung und Größe unterscheiden (siehe Exkurs). Dazu gehören sich von der Zelloberfläche abschnürende ,,shedding“ Mikrovesikel, dem endosomalen System entstammende, sezernierte Exosomen und apoptotische
Körper (Raposo und Stoorvogel 2013). Letztere werden während der Apoptose frei, die anderen Vesikeltypen werden von gesunden Zellen abgegeben. Ein Gemisch der extrazellulären Vesikel findet man in sämtlichen Körperflüssigkeiten, wie z.B. in Plasma, Urin oder auch in der Cerebrospinalflüssigkeit (CSF). Aufgrund ihres zelltypspezifischen Inhalts besitzen sie großes Potenzial für die Entwicklung von Anwendungen in der nicht-invasiven Diagnostik. Eine Revolution bei der Erfor- schung von extrazellulären Vesikeln war die Entdeckung von Ribonukleinsäuren in der Vesikelfracht und der Nachweis, dass diese RNA-Moleküle nach Aufnahme durch Empfängerzellen dort ihre Wirkung entfalten. So führt die Übertragung von mRNA zur Neusynthese von (möglicherweise zellfremden) Proteinen, wohingegen miRNAs die Produktion bestimmter zelleigener Proteine unterbinden. Durch den horizontalen Transfer von genetischer Information lassen sich ausgeprägte 
phänotypische Effekte in den Zielzellen hervorrufen.

Alle Typen von neuralen Zellen scheinen grundsätzlich in der Lage, verschiedene Arten der extrazellulären Vesikel zu generieren. Diese können im CSF und im Überstand von kultivierten Gliazellen und Neuronen dargestellt werden. Inwieweit solche Vesikel die Kommunikation zwischen Zellen im ZNS prägen, ist zum jetzigen Zeitpunkt sicher nur rudimentär verstanden. Am besten charakterisiert ist die Rolle von Exosomen bei der wechselseitigen Interaktion von Oligodendrozyten und Neuronen. Dabei stimulieren elektrisch aktive Neurone die Sekretion von oligodendroglialen Exosomen, die im Gegenzug von den Neuronen internalisiert werden und zu verbesserter Resistenz der Neurone gegen Stressfaktoren beitragen. Im Kontext der Komplexität des ZNS eröffnet die vesikelabhängige Zellkommunikation interessante und vielseitige Perspektiven.

\section{Allgemeine biologische Funktionen}

Seit ihrer Entdeckung vor ca. 30 Jahren wurden den extrazellulären Vesikeln zahlreiche Funktionen zugeordnet. Eine der ersten beschriebenen Funktionen war, dass unreife rote Blutkörperchen, die Retikulozyten, während der Zellreifung nicht mehr benötigte Membranproteine über Exosomenabgabe eliminieren. Somit können Zellen überschüssige zelluläre Komponenten freisetzen und dadurch eine Überlastung des intrazellulären Recyclingsystems vermeiden. Schon lange ist bekannt, dass von Thrombozyten gebildete Mikrovesikel eine wichtige Rolle bei der Blutgerinnung spielen. Die Erforschung der Bedeutung von extrazellulären Vesikeln für den Informationsaustausch zwischen Zellen hat ihren Ursprung vorwiegend in der Immunologie und der Tumorbiologie (Bobrie et al. 2011). Im Immunsystem geben Antigen-präsentierende Zellen und B-Zellen Exosomen ab, die MHC-Klasse II-Moleküle tragen und TZellen stimulieren können, was eine Rolle bei der adaptiven Immunantwort nahe legt. Tumorzellen sezernieren verhältnismäßig große Mengen von Mikrovesikeln und Exosomen, die recht heterogen in Zusammensetzung und Funktion erscheinen. Sie können zum einen durch Interaktion mit Antigen-präsentierenden Zellen tumorsuppressive Immunantworten auslösen, zum anderen jedoch durch Wirkung auf die Tu- mormikroumgebung das Tumorwachstum und die Metastasierung begünstigen. Tumorvesikel zirkulieren in der Blutbahn und können prognostisch relevante Information zu Tumorprogression und Therapieerfolgen im Rahmen einer nicht-invasiven Diagnostik liefern. Dies gilt auch für Mikrovesikel und Exosomen aggressiver Hirntumore. Tatsächlich können Vesikel, die Glioblastomen entstammen, in einigen Fällen die Bluthirnschranke passieren und in den Vesikeln enthaltene spezifische Tumormarker in der Zirkulation nachgewiesen werden.

Exosomen sind wohl auch an grundlegenden Entwicklungsprozessen beteiligt, z.B. indem sie Morphogene verbreiten (Gross und Boutros 2013). Exosomen von Drosophila- und humanen Zellen tragen das Morphogen Wnt und aktivieren Wnt-Signalwege in Zielzellen. Generell scheint die Kommunikation über Vesikel ein evolutionär konservierter Vorgang zu sein, der von Bakterien, Pilzen, Pflanzen, Invertebraten und Vertebraten gleichermaßen genutzt wird. Einzellige Parasiten, wie Leishmanien, Trypanosomen und Plasmodien, benutzen Exosomen zur Kommunikation mit Wirtszellen und zum Transfer von Virulenzfaktoren.

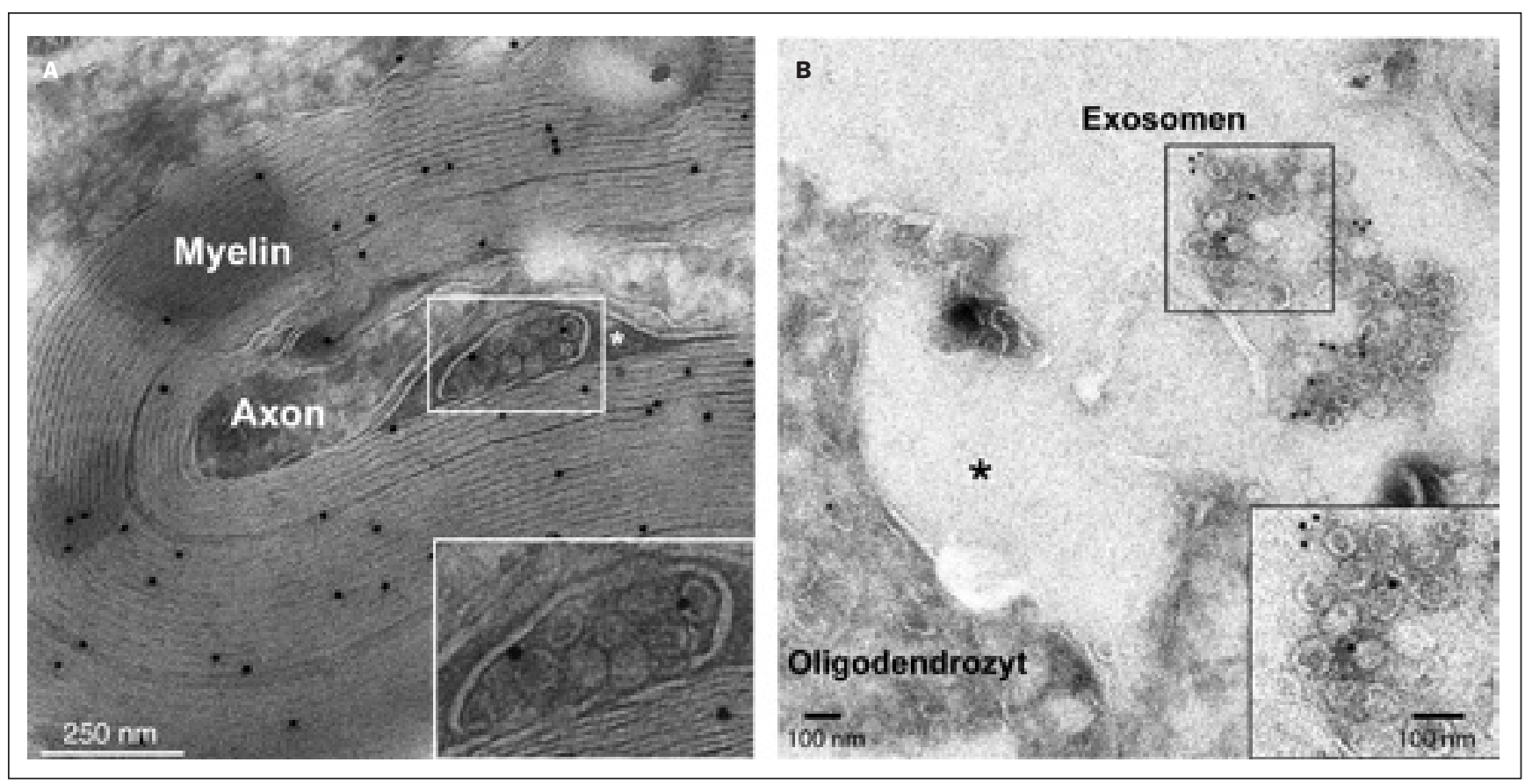

Abb. 1: Elektronenmikroskopische Darstellung von Exosomen und ihrer zellulären Vorstufe, den Multivesikulären Endosomen. A) Der Querschnitt einer myelinisierten Nervenfaser zeigt ein Multivesikuläres Endosom (vergrößert in der Box), das in direkter Nachbarschaft zum Axon liegt und nach Fusion mit der Plasmamembran die intraluminalen Vesikel als Exosomen freigeben kann. Multivesikuläre Endosomen befinden sich gehäuft an Stellen gegenüber dem Axon. B) Exosomen, die von kultivierten Oligodendrozyten in den extrazellulären Raum abgegeben wurden (Stern markiert die potenzielle Fusionsstelle des MVE). Oligodendrogliale Exosomen haben ein sehr homogenes Erscheinungsbild und sind im Durchmesser kleiner als $100 \mathrm{~nm}$ (nach Frühbeis et al., 2012 und 2013b.). 


\section{Bedeutung von Exosomen und Mikrovesikeln im Nervensystem}

Im Nervensystem gibt es Hinweise auf einen Transfer von Vesikeln sowohl unter Neuronen als auch zwischen Gliazellen und Neuronen (Frühbeis et al. 2013a und Referenzen darin). Der Transfer neuronaler Exosomen wird hauptsächlich im Kontext von synaptischer Plastizität diskutiert. Die besten Evidenzen dafür stammen aus der Fruchtfliege. An der neuromuskulären Verbindung von Drosophila-Larven werden Exosomen genutzt, um das Wnt-Signalprotein zusammen mit seinem Transportrezeptorprotein Evi/Wls sowie Synaptotagmin 4 von der Präsynapse zur postsynaptischen Muskelzelle zu übertragen. Dieser Transfer bewirkt retrograde Signale, welche die Ausdehnung der Synapse kontrollieren. Auch im Nervensystem von Säugetieren wird eine Beteiligung von Exosomen an synaptischen Prozessen vorgeschlagen. Kultivierte kortikale Neurone setzen Exosomen in Abhängigkeit von glutamaterger Aktivität frei, die unter anderem AMPA-Rezeptoruntereinheiten beinhalten. Dies führt möglicherweise zur Reduktion der Zahl funktionaler postsynaptischer AMPA-Rezeptoren und zur Verminderung der Erregbarkeit des postsynaptischen Neurons. Die aktivitätsabhängige Freisetzung von Exosomen aus postsynaptischen, somato-dendritischen Bereichen der Neurone könnte der Regulation der Signalübertragungsstärke dienen und so einen Mechanismus von synaptischer Plastizität darstellen (Chivet et al. 2013). Folglich scheinen Exosomen sowohl bei Invertebraten als auch bei Vertebraten eine Rolle bei der transsynaptischen Kommunikation zu spielen.

Neben Neuronen sezernieren auch Mikroglia und alle Arten von Makroglia Exosomen oder Mikrovesikel. Mikroglia sind im Gehirn ansässige makrophagenartige Zellen, die für Gewebehomöostase sorgen und in aktiviertem Zustand an Immunabwehr- und Gewebereparaturprozessen beteiligt sind. Astrozyten sind Teil der Blut-Hirn-Schranke, kontrollieren das extrazelluläre Ionengleichgewicht und sind zusammen mit den Mikroglia an der Reparatur und Vernarbung nach ZNSVerletzungen beteiligt. Sowohl Mikroglia als auch Astrozyten schnüren Mikrovesikel in Antwort auf purinerge Signale nach Aktivierung des $\mathrm{P}_{2} \mathrm{X}_{7}$-Rezeptors von der Plasmamembran ab. Die von Mikroglia stammenden Mikrovesikel verstärken die Neurotransmission und beeinflussen damit die synaptische Aktivität von Neuronen (Prada et al. 2013). Interessanterweise enthalten Mikroglia-Mikrovesikel das proinflammatorische Zytokin Interleukin-1 $\beta$ und tragen wahrscheinlich zur Verstärkung und Verbreitung von Entzündungsreaktionen im ZNS bei. Im gesunden Gehirn sind verschiedene Vesikeltypen aller Gliazellen im CSF detektierbar. Im Fall von Entzündungen, z.B. bei Multipler Sklerose, nimmt die Menge an mikroglialen Mikrovesikeln erheblich zu.

Astrozyten generieren eine sehr heterogene Population von extrazellulären Vesikeln, deren Funktionen weniger gut erforscht sind. Sie enthalten neben verschiedenen protektiven oder trophischen Substanzen Enzyme des Energiestoffwechsels und zum Teil gar mitochondriale Elemente. Sie wurden daher im Zusammenhang mit Schutzfunktionen oder metabolischer Unterstützung für Neurone diskutiert.

\section{Oligodendrogliale Exosomen und Axon-Glia-Kommunikation}

Oligodendrozyten bilden die Myelinscheide und ermöglichen damit die saltatorische Erregungsleitung. Die Umhüllung der
Axone mit einer mehrschichtigen Myelinmembran führt zur Bildung einer komplexen funktionellen Einheit und basiert auf kontinuierlichem wechselseitigen Signalaustausch zwischen beiden Zelltypen. So ist zur Aufrechterhaltung der axonalen Integrität Unterstützung durch den Oligodendrozyten notwendig. Fällt diese externe Versorgung weg, degenerieren die Axone (Nave 2010). Diese Versorgung scheint durch den Transfer von energiereichen Substraten über spezielle Transportkanäle zum Axon vermittelt zu werden. Nach der Myelinisierung gewinnen Oligodendrozyten ihre Energie vorwiegend aus der Glykolyse und könnten das dabei entstehende Laktat dem „energiehungrigen“ Axon zur Verfügung stellen (Morrison et al. 2013). Eine zusätzliche Rolle bei der Erhaltung der Axone spielt möglicherweise auch der Transfer von Exosomen von Oligodendrozyten zum Axon (Frühbeis et al. 2013b; Lewis 2013).

Oligodendrozyten sekretieren Exosomen durch Fusion von Multivesikulären Endosomen (MVEs) mit der Plasmamembran (Abbildung 1, Frühbeis et al. 2013a). Neben den typischen Exosomenassoziierten Proteinen und RNA enthalten von Oligodendrozyten stammende Exosomen Myelinproteine, wie z.B. PLP und CNP, sowie verschiedene Enzyme. Ruhende Mikroglia können diese Exosomen mittels Makropinozytose internalisieren und degradieren, ohne immunologische oder inflammatorische Antworten zu erzeugen. Des Weiteren können oligodendrogliale Exosomen auch autokrin wirken und die Myelinsynthese inhibieren. Über ihre Wirkung auf Neurone jedoch war bisher nichts bekannt. Interessanterweise findet man die

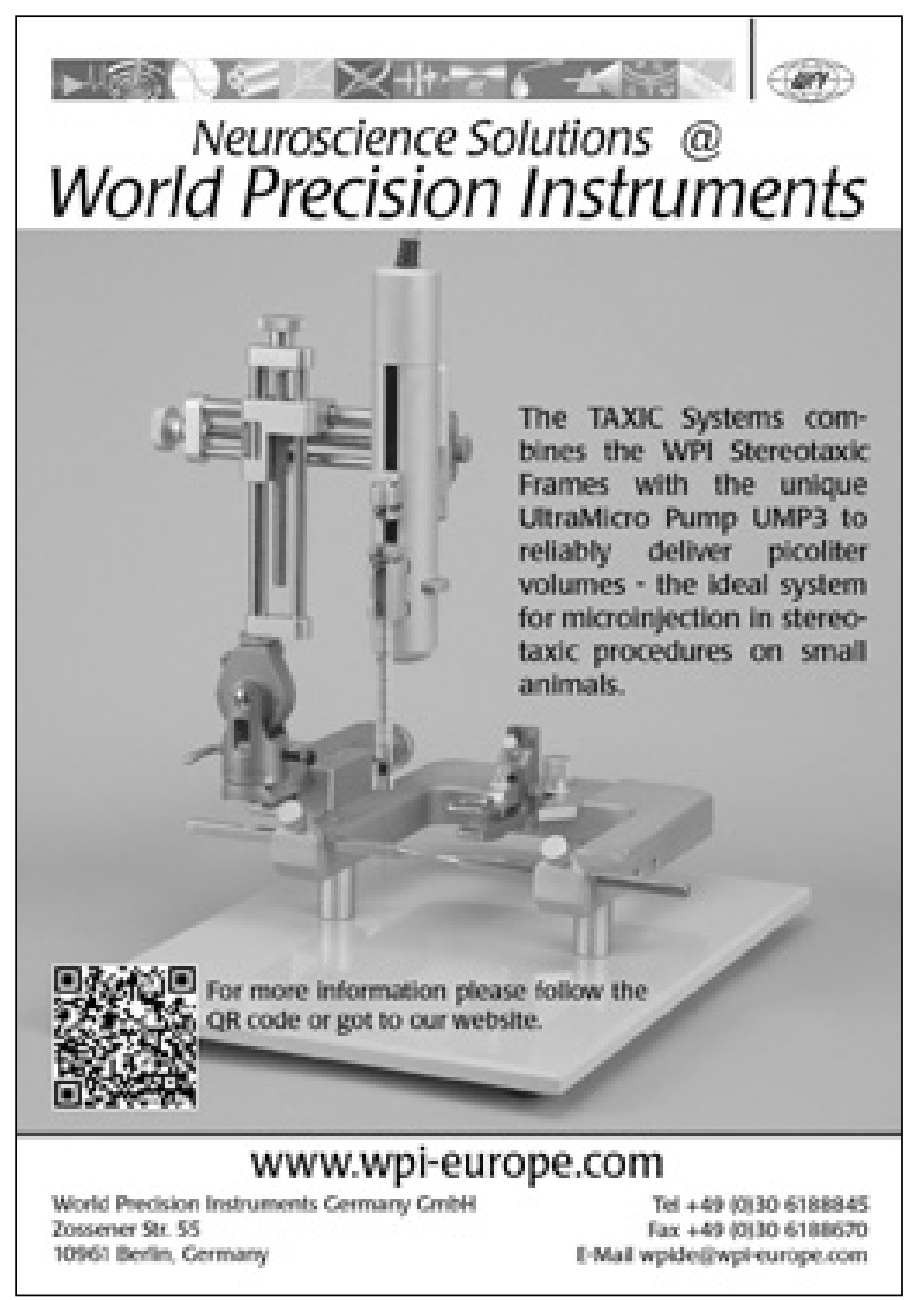




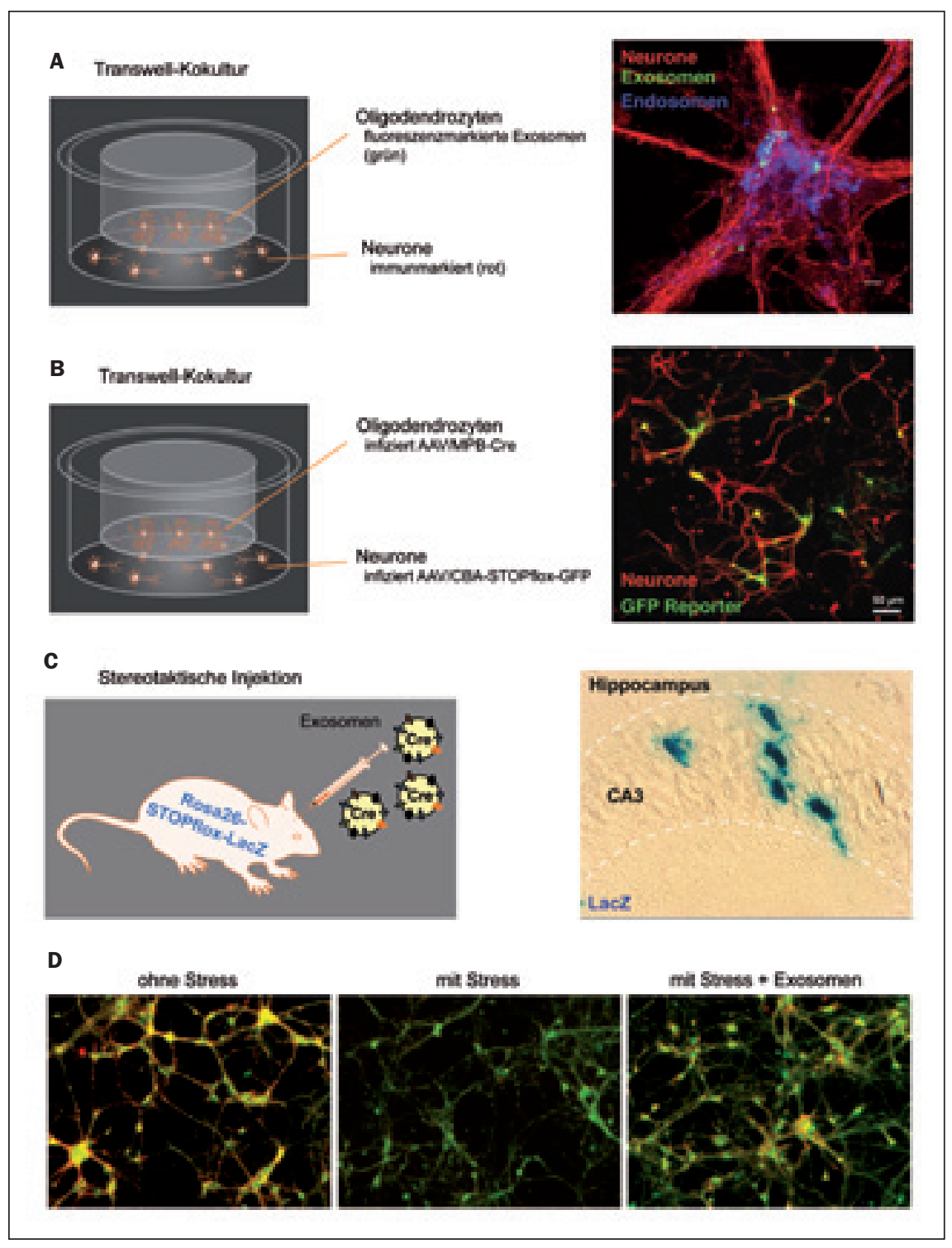

Abb. 2: Aufnahme und Verwertung von glialen Exosomen durch Neurone. A, B) Der Exosomentransfer von Oligodendrozyten zu Neuronen kann im Transwell-Kultursystem dargestellt werden, wobei kein direkter Kontakt zwischen beiden Zelltypen besteht, jedoch Austausch über das Kulturmedium stattfinden kann (schematische Darstellung links). Oligodendrozyten wachsen auf einer Filtermembran, die Poren einer definierten Größe besitzt und Exosomen passieren lässt. Neurone sind in der Kulturkammer darunter platziert. Die spezifische Markierung der oligodendroglialen Exosomen mit einem Fluoreszenzfarbstoff (A) oder mit einem funktionalen Enzym (B) erlaubt dann den Nachweis des Transfers zu den Neuronen (Darstellung immungefärbter Neurone rechts). B) Die Infektion von Oligodendrozyten mit einem viralen Vektor, der die Cre-Rekombinase trägt, führt zum Exosomen-vermittelten Transfer von Cre zu Zielneuronen und zur Cre-vermittelten Rekombination eines dort präsenten GFP-Reportergens. Expression von GFP in Zielneuronen zeigt den glia-neuronalen Transfer und darüber hinaus die funktionale Gewinnung der exosomalen Inhaltsstoffe durch die Zielneurone an. C) Stereotaktische Injektion von Cre-markierten oligodendroglialen Exosomen ins Gehirn von transgenen Mäusen, die in allen Zellen ein Cre-Reportergen tragen, bewirkt die Expression des Reportergens in Neuronen (z.B. im Hippocampus, Bild rechts) und beweist, dass die Aufnahme der injizierten glialen Exosomen durch Neurone im intakten Gewebe stattfinden kann. D) Färbung von Neuronen mit einem Fluoreszenzfarbstoff, der die Fähigkeit von Mitochondrien zur Energieproduktion anzeigt. Neurone, die durch Entzug von Nährstoffen unter Stress gesetzt werden, besitzen ein geringes mitochondriales Membranpotenzial (mittleres Bild, grün). Die Anwesenheit von Exosomen stützt das mitochondriale Potenzial (rechtes Bild, rot bzw. gelb) und schützt damit die Neurone (Adaptiert von Frühbeis et al., 2013b). zellulären Vorstufen der Exosomen, die MVEs, in myelinisierten Nervenfasern vorwiegend in der innersten, nicht kompaktierten Windung der Myelinmembran in räumlicher Nähe zum Axon. So stellte sich die Frage, ob Exosomen eventuell in den periaxonalen Raum abgegeben werden und an Axon-Glia-Interaktion beteiligt sind. Tatsächlich nehmen Neurone durch Neurotransmittersignale Einfluss auf die Exosomensekretion der Oligodendrozyten. Die elektrische Aktivität von exzitatorischen Neuronen führt zur Freisetzung des Neurotransmitters Glutamat. Dieses aktiviert oligodendrogliale ionotrope Glutamatrezeptoren, die den Einstrom von Kalziumionen vermitteln und so die Freisetzung von Exosomen stimulieren. Dabei scheinen Rezeptoren des NMDA-Typs von entscheidender Bedeutung zu sein. Die abgegebenen Exosomen werden von Neuronen über Clathrin- und Dynaminabhängige Endozytose aufgenommen (Abbildung 2). Der Aufnahmeprozess kann nicht nur in den somatodendritischen sondern auch in den axonalen Regionen der Nervenzelle stattfinden und scheint zudem selektiv zu erfolgen, da Astrozyten und Oligodendrozyten diese Exosomen nur in sehr geringem Maße internalisieren. Eine wichtige Frage ist, ob die Fracht der Exosomen nach der Aufnahme von den Zielneuronen genutzt wird. Tatsächlich ist dies der Fall und konnte durch folgende experimentelle Strategie nachgewiesen werden: Nach ektopischer Expression von Cre-Rekombinase in Oligodendozyten wird Cre in Exosomen verpackt und ist in einer Kokultur nach Transfer zu Zielneuronen in der Lage, dort ein Cre-induziertes Reportergen anzuschalten. Injiziert man Cre-enthaltende Exosomen ins Gehirn von Reportermäusen, wird auch hier der Reporter in Neuronen aktiviert. So ist ein wichtiger Beweis erbracht, dass der Transfer von oligodendroglialen Exosomen nicht nur in vitro, sondern auch im Gehirn stattfinden kann. Dies wird von Beobachtungen unterstützt, dass auch bei Mäusen, in denen Cre unter Kontrolle eines Oligodendrozyten-spezifischen Promoters steht, vereinzelt die Rekombination von Neuronen in verschiedenen Hirnregionen festzustellen ist.

Was bewirken nun aber die Exosomen in den neuronalen Zielzellen? Experimente mit kultivierten Neuronen zeigen, dass die Beigabe von oligodendroglialen Exosomen die metabolische Aktivität der Neurone insbesondere bei Nährstoffdefiziten oder oxidativem Stress verbessert. Auch im Peripheren Nervensystem (PNS) 
gibt es Evidenzen, dass myelinisierende Schwann-Zellen unterstützende Substanzen per Exosomen zu Axonen schicken, die sogar deren Regenerationsfähigkeit im Falle einer Verletzung erhöhen. Oligodendrogliale Exosomen scheinen wie „Care“-Pakete zu funktionieren und gemäß dem Prinzip „Lieferung auf Abruf“ neuroprotektive Eigenschaften zu übertragen (Abbildung 3). Besonders elektrisch aktive Neurone sind von Energiedefiziten und oxidativem Stress betroffen. Insofern erscheint es sinnvoll, die Exosomenabgabe an Neurotransmittersignale zu koppeln. Damit signalisieren aktive Neurone mit einer Art Hilferuf den Oligodendrozyten, dass sie externe Zufuhr von Biomolekülen benötigen. Die „Care“-Pakete in Form von Exosomen liefern dann Metabolite, protektive Proteine (z.B. Hitzeschockproteine), Glykolyseenzyme, mRNA und miRNA, welche vermutlich den Stoffwechsel des Axons unterstützen und dazu beitragen, die axonale Integrität aufrechtzuerhalten (Frühbeis et al. 2013b).

Die trophische Unterstützung von Neuronen durch die Oligodendrozyten offenbart sich in Knockout-Mäusen, denen die Gene der Myelinproteine CNP und PLP fehlen. Diese Tiere entwickeln axonale Schwellungen und eine sekundäre axonale Degeneration (Nave 2010). Da beide Proteine in oligodendroglialen Exosomen vorkommen, ist es durchaus möglich, dass ihre Funktion bei der glialen Unterstützung mit dem exosomalen Transfer von Substanzen zusammenhängt. Die Abwesenheit von PLP und CNP könnte beispielsweise die Freisetzung von Exosomen beeinflussen und damit die Versorgung der Axone mit trophischen Faktoren. Dieser Sachverhalt lässt sich durch Analyse der oligodendroglialen Exosomensekretion in PLP- und CNP-Knockout-Tieren aufklären. Außerdem stellt sich die Frage nach der Natur der funktionellen Komponenten, die die unterstützende Wirkung auf Neurone haben. Exosomen enthalten eine ganze Reihe potenziell protektiver Substanzen, wie z.B. stressmildernde Proteine und verschiedene RNA-Arten. Offen ist zum aktuellen Zeitpunkt auch, ob RNA-Transfer via Exosomen tatsächlich in lokaler Translation im Axon resultiert.

\section{Klinische Relevanz}

Mit der Strategie eines Trojanischen Pferdes könnte die vesikelabhängige Übertragung von Proteinen die Ausbreitung von ZNS-Pathologien begünstigen. Pathogene Proteine, wie z.B. Prionen, Amyloid- $\beta$, Superoxid-

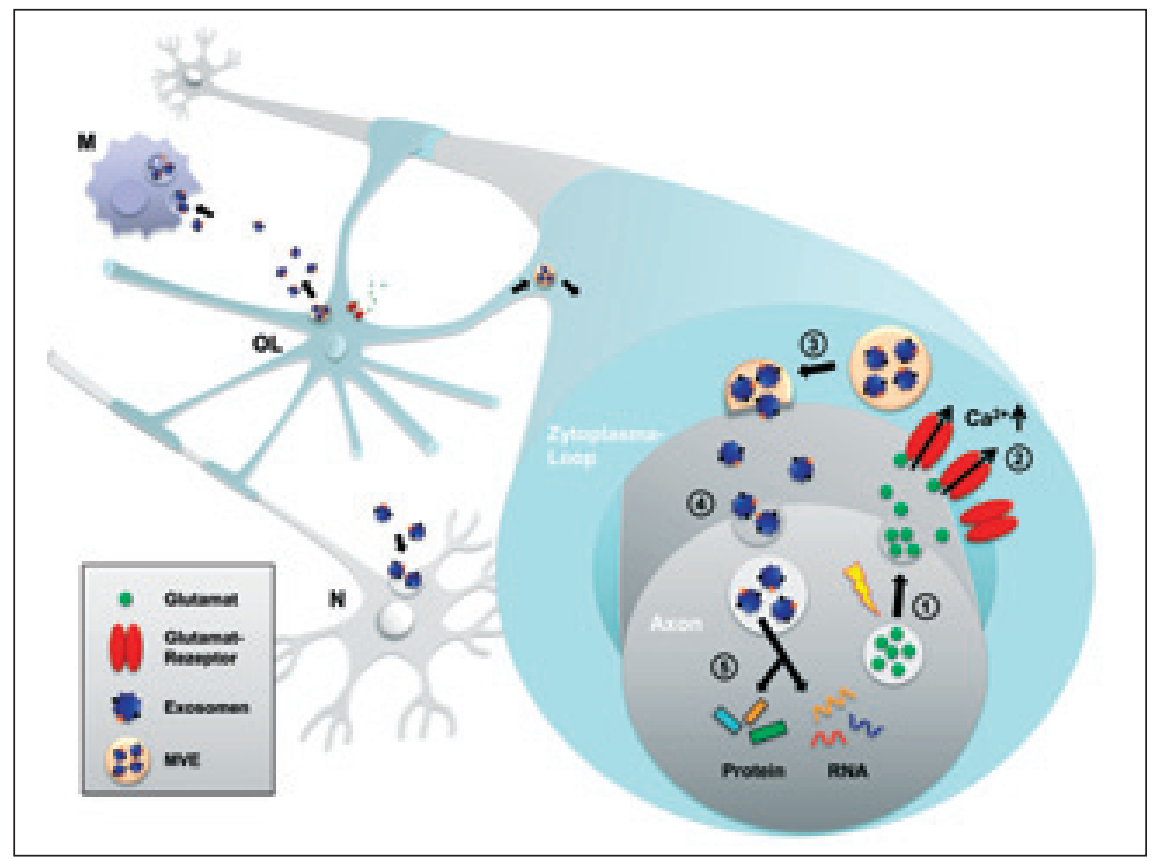

Abb. 3: Die regulierte Abgabe von Exosomen durch Oligodendrozyten und ihre Rolle bei der Axon-Glia Interaktion. Elektrisch aktive Axone setzen den Neurotransmitter Glutamat frei (1), was einen Kalziumeinstrom durch oligodendrogliale Glutamatrezeptoren bewirkt (2). Der Anstieg der intrazellulären Kalziumkonzentration löst die Exosomenabgabe der Oligodendrozyten (OL) aus (3). Neurone $(\mathrm{N})$ internalisieren die Exosomen an Axonen und Zellkörpern (4) und verwenden die Protein- und RNA-Fracht (5). Auch Mikroglia (M) nehmen oligodendrogliale Exosomen auf (Frühbeis et al. 2013b).

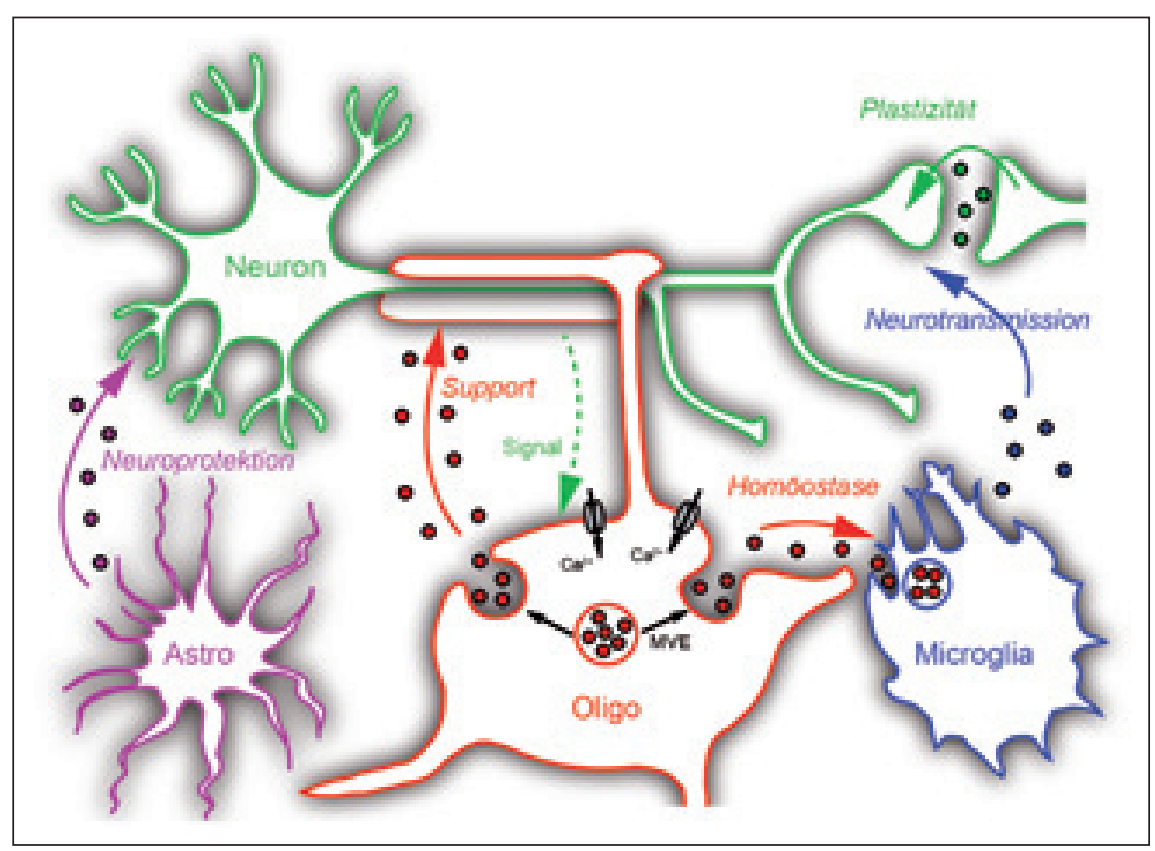

Abb. 4: Extrazelluläre Vesikel und Zellkommunikation im Nervensystem. Die Zellen des Nervensystems geben verschiedene Typen von extrazellulären Vesikeln ab. Neurone setzen Exosomen frei, die synaptische Plastizität beeinflussen können. Mikroglia modulieren Neurotransmission über Mikrovesikel. Astrozyten geben Exosomen ab, die neuroprotektive Fracht tragen. Oligodendrozyten sezernieren auf neuronale Signale hin Exosomen. Nach Aufnahme durch die Neurone unterstützen diese Exosomen die Neurone metabolisch. Mikroglia degradieren oligodendrogliale Exosomen (nach Frühbeis et al. 2012). 
Dismutase (SOD1) und Tau, werden in Verbindung mit Exosomen aus Zellen freigesetzt. Alle diese Proteine haben die Eigenschaft die Bildung von Aggregaten zu begünstigen, die von Zellen nicht degradiert werden können. Von Zielzellen aufgenommen könnten sich diese pathogenen Proteine mithilfe von Exosomen nach der Art von infektiösen Agenzien im Nervengewebe verbreiten (Schneider und Simons 2012). Die exosomenabhängigen Verbreitungsprozesse der pathogenen Proteine im Gewebe sind jedoch bisher nicht gut verstanden. Schon eher durchschaut man die tumorfördernde Funktion von Mikrovesikeln/Exosomen, die von Gliomazellen in ihre Umgebung abgegeben werden. Sie tragen die onkogene Variante des EGF-Rezeptors EGFRvIII, verschiedene Arten von RNA, sowie Blutgefäß bildende Faktoren. Damit sind sie in der Lage, die Transformation von Zielzellen zu fördern und die Wachstumsbedingungen des Tumors zu verbessern.

Eine Entzündung im ZNS, wie sie z.B. die Multiple Sklerose darstellt, führt zu einer erhöhten Zahl von Vesikeln im CSF, die größtenteils von Mikrogliazellen stammen. Die relative Präsenz dieser Vesikel scheint mit den klinischen Symptomen zu korrelieren, und sie üben möglicherweise einen pro-inflammatorischen Effekt aus. In jedem Fall stellen die im CSF präsenten Vesikel (sowie ihr spezifischer Inhalt) ein potenzielles Mittel für die Diagnostik von ZNS-Erkrankungen dar. Allerdings ist die Liquorentnahme eher eine invasive Prozedur. In welchem Maße dem ZNS entstammende Vesikel tatsächlich im Plasma nachweisbar sind, ist bisher nicht ausreichend erforscht.

Exosomen könnten auch als therapeutische Vehikel für den Transfer von Wirkstoffen in das ZNS dienlich sein. Biotechnologisch modifizierte Exosomen aus dendritischen Zellen sind nach systemischer Injektion in der Lage, die Bluthirnschranke zu passieren und die Wirkung einer ektopischen regulatorischen RNA im ZNS zu erzielen. Der Tropismus kann dabei durch definierte Rezeptormoleküle in der Exosomenhülle bestimmt werden. Mäusen nasal applizierte, mit antiinflammatorischen Substanzen beladene Exosomen gelangen auf bisher nicht weiter charakterisiertem Wege ins Gehirn. Sie werden dort von Mikroglia aufgenommen und sind in der Lage, Neuroinflammation $\mathrm{zu}$ vermindern. Auch wenn noch viel Grundlagenarbeit zur Biologie von Exosomen und Mikrovesikeln zu leisten ist, besteht die Aussicht, Exosomen vielleicht eines Tages als „Cure“-Pakete zur Therapie von ZNS-Erkrankungen einzusetzen.

\section{Fazit}

In der aktuellen Forschung entwickeln sich extrazelluläre Vesikel zu bedeutenden Akteuren im ZNS (Abbildung 4). Diese Vesikel beeinflussen Neurotransmission, unterstützen Neurone, verbreiten pathogene Faktoren und begünstigen Entzündungen. Nach der Devise ,Lieferung auf Abruf" geben Oligodendrozyten Exosomen auf neuronale Signale hin ab, die protektive Substanzen zu Neuronen transportieren und möglicherweise zur axonalen Integrität beitragen. Viele der Grundlagenerkenntnisse basieren auf Zellkulturexperimenten, deren Bedeutung sich in Zukunft in vivo, z.B. in genetischen Mausmodellen, validieren muss. Das Forschungsfeld der vesikelbasierten Zellkommunikation steht am Beginn und lässt spannende Entwicklungen in den Neurowissenschaften erwarten.

\section{Danksagung}

Die Autoren danken Frau Prof. Jacqueline Trotter für die Unterstützung und allen Mitgliedern der Abteilung Molekulare Zellbiologie für die konstruktive Diskussion.

Unsere Arbeit wird von der DFG (KR3668/1-1) und dem inneruniversitären Forschungsförderprogramm der Johannes Gutenberg Universität Mainz unterstützt.

\section{Literatur}

Bobrie, A. und Colombo, M., et al. (2011): Exosome secretion: molecular mechanisms and roles in immune responses. Traffic 12 (12): 1659-1668.

Chivet, M., Javalet, C. et al. (2013): Exosomes as a novel way of interneuronal communication. Biochem Soc Trans 41 (1): 241-244.

Frühbeis, C., Fröhlich, D. et al. (2012): Emerging roles of exosomes in neuron-glia communication. Front Physiol (3): 119.

Frühbeis, C., Fröhlich, D. et al. (2013b): Neurotransmitter-triggered transfer of exosomes mediates oligodendrocyte-neuron communication. PLoS biology 11 (7): e1001604.

Frühbeis, C., Fröhlich, D. et al. (2013a): Extracellular vesicles as mediators of neuron-glia communication. Front. Cell. Neurosci. 7 (182).

Gross, J. C. und Boutros, M. (2013): Secretion and extracellular space travel of Wnt proteins. Current opinion in genetics \& development 23 (4): 385-390.

Lewis, S. (2013): Glia: Transporting cargo from A to B. Nature reviews. Neuroscience 14 (9): 589.
Morrison, B. M., Lee, Y. et al. (2013): Oligodendroglia: metabolic supporters of axons. Trends Cell Biol. Aug 27, epub ahead of print.

Nave, K. A. (2010): Myelination and support of axonal integrity by glia. Nature 468 (7321): 244-252.

Prada, I., Furlan, R. et al. (2013): Classical and unconventional pathways of vesicular release in microglia. Glia 61 (7): 1003-1017.

Raposo, G. und Stoorvogel, W. (2013): Extracellular vesicles: exosomes, microvesicles and friends. The Journal of cell biology 200 (4): 373-383.

Schneider, A. und Simons, M. (2013): Exosomes: vesicular carriers for intercellular communication in neurodegenerative disorders. Cell and tissue research. 352 (1): 33-47.

Thery, C. (2011): Exosomes: secreted vesicles and intercellular communications. F1000 Biol Rep 3: 15.

\section{Kurzbiografien}

Eva-Maria Krämer-Albers: Studium der Biologie in Heidelberg. 1997 Promotion am Institut für Neurobiologie der Universität Heidelberg. 1998-2000 Postdoc am Zentrum für Molekulare Biologie Heidelberg und am Max-Planck-Institut für experimentelle Medizin in Göttingen (Abt. Neurogenetik). Seit 2001 Wissenschaftlerin in der Molekularen Zellbiologie der Universität Mainz. Mitglied im Forschungszentrum Translationale Neurowissenschaften der Universität Mainz.

Carsten Frühbeis studierte Biologie an den Universitäten Mainz und Heidelberg und promovierte 2007 am Institut für Molekulare Biowissenschaften der Universität Frankfurt/M. Seitdem arbeitet er als Postdoc in der Molekularen Zellbiologie der Universität Mainz.

\section{Korrespondenzadresse}

Dr. Eva-Maria Krämer-Albers

Molekulare Zellbiologie

Johannes Gutenberg - Universität Mainz 55099 Mainz

E-Mail: alberse@uni-mainz.de 


\section{Springer Spektrum}

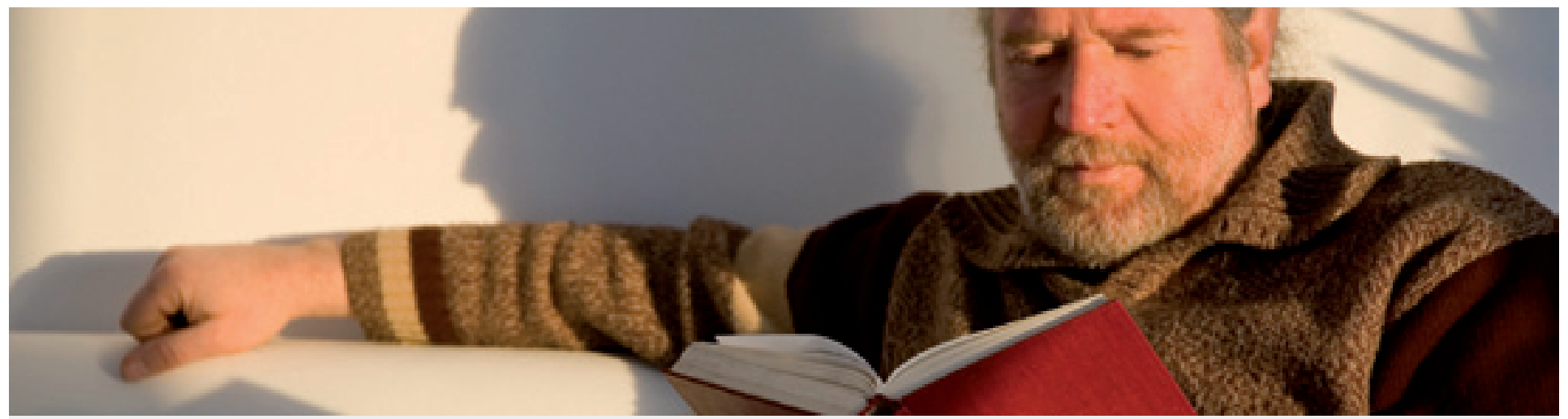

\section{Spektrum Sachbücher Aktuelle Neuerscheinungen \& Highlights}

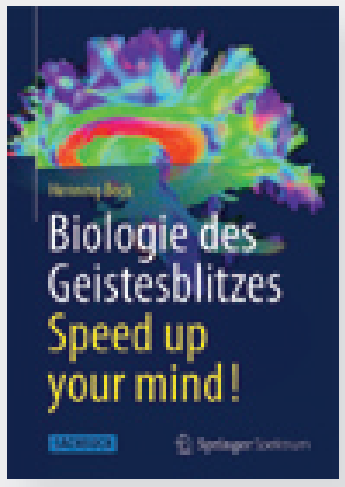

2013, 243 S. 63 Abb. in Farbe. Brosch. ISBN 978-3-642-36532-4

$€$ (D) $14,99|€(A) 15,37|{ }^{*}$ sFr 16,50

Henning Beck

Biologie des Geistesblitzes

Humorvoll und fachlich fundiert erklärt der deutsche Science-Slam-Meister 2012 Henning Beck anhand von Beispielen aus dem Alltag, was die Hirnforschung zum Thema „Kreativität" zu sagen hat, was ein sogennter Geistesblitz überhaupt ist: wie dieser entsteht. Dabei zeigt er nicht nur, wie und woran auf diesem Gebiet geforscht wird, sondern macht die kreativen Prozesse im Gehirn verständlich. Ein höchst stimulierendes Lesevergnügen!

$\epsilon$ (D) sind gebundene Ladenpreise in Deutschland und enthalten $7 \%$ MwSt. $€$ (A) sind gebundene Ladenpreise in Österreich und enthalten $10 \%$ MwSt. Die mit * gekennzeichneten Preise sind unverbindliche Preisempfehlungen und enthalten die landesübliche MwSt. Preisänderungen und Irrtümer vorbehalten.

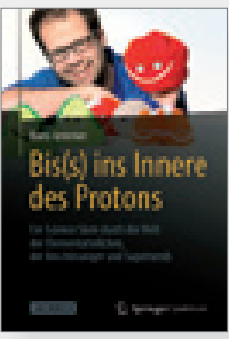

Boris Lemmer

Bis(s) ins Innere des Protons

Science Slam-Meister Boris Lemmer über die Weit der Teilchenphysik.

"Bei vielen seiner witzigen Ideen, Physik humorvoll zu erklären, war ich echt sauer. Ich dachte mir: Warum zum Teufel bin ich da nicht draufgekommen ..."Vince Ebert

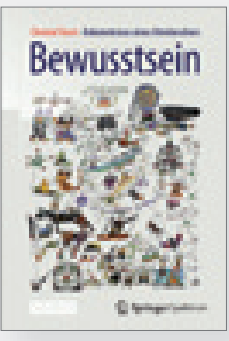

2013, 332 S. Geb. ISBN 978-3-642-34770-2 $€(D) 24,95 \mid €(A) 25,65$ ${ }^{*} \mathrm{sFr} 31,50$

Christof Koch

Bewusstsein

Autobiographische Überlegungen eines renommierten Hirnforschers zu Fragen wie z. B. „Wie erleben wir Gefühle, wie Bewusstsein?" Koch vertritt die These, dass sich mit naturwissenschaftlichen Methoden erklären lässt, wie Gefühle entstehen.

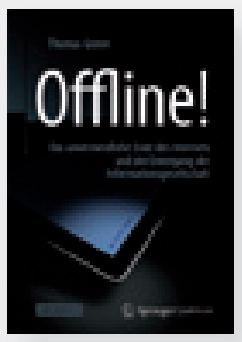

2013. 264 S. 10 Abb. Geb. ISBN 978-3-642-37736-5 $€$ (D) $19,99 \mid €(A) 20,51$ *sFr 25,00

Thomas Grüter Offline!

Das scheinbar so robuste Internet wird in wenigen Jahrzehnten zusammenbrechen. Die Informationsgesellschaft wird davon mitgerissen - möglicherweise unwiderruflich. Das ist die provozierende These des Sachbuchautors Thomas Grüter.

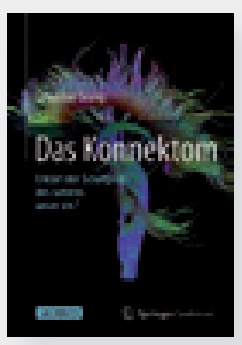

2013, 321 S. 53 Abb. Geb. ISBN 978-3-642-34294-3 $€$ (D) $24,99 \mid €(A) 25,69$ *sFr 31,50

Sebastian Seung

Das Konnektom - Erklärt der Schaltplan des Gehirns unser Ich?

Das Buch beschreibt die erstaunlichen technischen Fortschritte, die uns bald helfen werden, unser Gehirn zu kartieren und damit den Schaltplan des Geistes sichtbar zu machen. Willkommen in der Zukunft der Neurowissenschaften! 


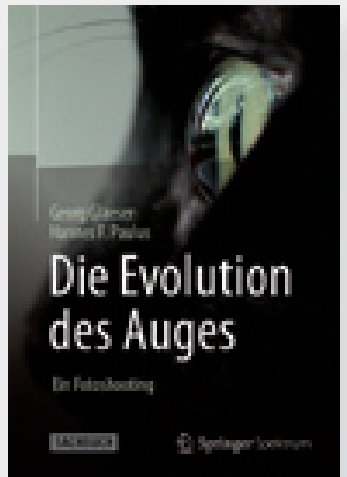

2014, XVI, 214 S. Geb.

ISBN 978-3-642-37775-4

$€$ (D) $24,99|€(A) 25,69|$ *sFr 31,50

Georg Glaeser, Hannes F. Paulus

Die Evolution des Auges -

\section{Ein Fotoshooting}

Spektakuläre, großformatige Fotos auf Doppelseiten mit einem Erklärungstext machen die Leser neugierig auf das, was die Evolution im Bereich der Augen hervorgebracht hat: unabhängig bewegbare Augen, Stielaugen, Pigmentbecher, Lochkameraaugen, Komplexaugen, ...

Das Buch kann in beliebiger Reihenfolge, Doppelseite für Doppelseite, gelesen werden. Querverweise sorgen für bequemes Umspringen auf andere Doppelseiten. Die Textpassagen sind zumeist - abgesehen von der fundierten Einleitung in jedem der 10 Kapitel unabhängig voneinander und besprechen besondere Highlights im evolutionären Prozess. Ergänzt wird die

Doppelseite mit Literaturhinweisen und Verweisen auf instruktive Internet-Seiten.

Stichworte zum Inhalt: Auge - Augensprache - Augentypen - Evolution - Facettenaugen - Farbsehen - Homologie Linsenaugen - Lochkameraauge - Optik - Pax-Gene - Retina - Räumliches Sehen - Sehen - Sehen unter Wasser

$€$ (D) sind gebundene Ladenpreise in Deutschland und enthalten $7 \%$ MwSt. $€$ (A) sind gebundene Ladenpreise in Österreich und enthalten $10 \%$ MwSt. Die mit * gekennzeichneten Preise sind unverbindliche Preisempfehlungen und enthalten die landesübliche MwSt. Preisänderungen und Irrtümer vorbehalten.

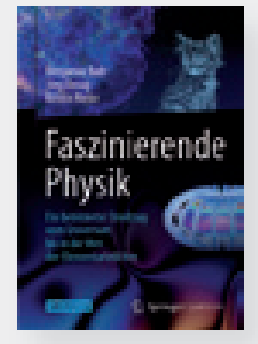

2013, 326 S., 680 Abb. in Farbe. Geb. ISBN 978-3-642-37811-9

$€$ (D) $29,99|€(A) 30,83|{ }^{*} \mathrm{sFr} 37,50$

B. Bahr, J. Resag, K. Riebe

Faszinierende Physik

Dieses reich illustrierte Lesebuch bietet einen packenden Streifzug durch die spannendsten und aktuellsten Themen der modernen Physik! Auf jeweils einer Doppelseite wird dem Leser dabei mit vielen Bildern ein kompakter und klar verständlicher Einstieg - fast ganz ohne Formeln - in jeweils ein Thema und den Stand der Forschung geboten. Mit weltweit einmaligen Abbildungen!

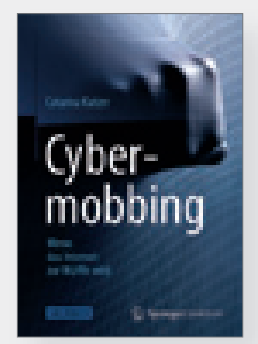

2014, X, 239 S. 10 Abb. Geb.

ISBN 978-3-642-37671-9

$€$ (D) $19,99|€(A) 20,55|{ }^{*}$ sFr 25,00

Catarina Katzer

\section{Cybermobbing - Wenn das Internet zur W@ffe wird}

Die international anerkannte Cyberpsychologin Catarina Katzer lässt in ihrem Buch einen spannenden Diskurs um das Cyberlife unserer Kinder aufleben bzw. um das Leben im Netz, das geprägt ist von einem ständigen Wechselspiel zwischen Faszination und Gefahr. Sie Klärt anschaulich und fundiert über den Tatort Internet auf und gibt hilfreiche und wertvolle Tipps zur Prävention.

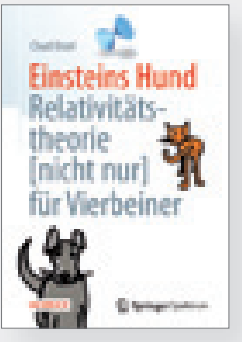

2013, 349 S. 43 Abb. Geb. ISBN 978-3-642-34758-0 $€$ (D) $19,99 \mid €$ (A) $20,55 \mid{ }^{*}$ sFr 25,00

Chad Orzel

Einsteins Hund

Begleiten Sie Physikprofessor Chad Orzel und seinen Hund Emmy auf eine neue Entdeckungsreise in die Welt der Physik! Nach „Schrödingers Hund“, der unterhaltsamen Einführung in die Quantenphysik, lernen Sie in diesem Buch nun die Relativitätstheorie mit anderen Augen kennen. Aus der Sicht eines Hundes werden Sie Einsteins Gedankengebäude viel leichter verstehen - zumindest haben Sie mehr Spaß, wenn es schwierig wird!

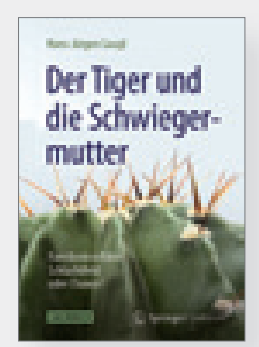

2013, X, 198 S. 10 Abb., Brosch. ISBN 978-3-642-38993-1 $€$ (D) $14,99 \mid €$ (A) 15,41 | * sFr 19,00

Hans-Jürgen Gaugl

Der Tiger und die Schwiegermutter

Der Autor lädt Sie mit diesem Buch ein, zahlreiche beispielhafte Schilderungen aus der Begegnung von Schwiegermüttern mit Schwiegerkindern zunächst zu beschmunzeln und anschließend aus Sicht des Konfliktmanagements auf Lösungsmöglichkeiten hin zu untersuchen. Haben Sie Schwierigkeiten mit Ihrer Schiegermutter? Dann lesen Sie dieses Buch!

\section{Einfach bestellen: SpringerDE-service@springer.com}




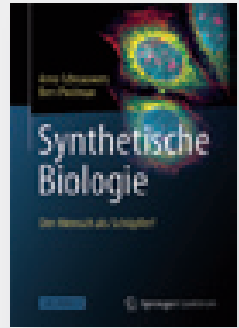

2013.215 S. 216 Abb. in Farbe. Brosch.

ISBN 978-3-642-34592-0 $€$ (D) $29,95|€(A) 30,79|$ ${ }^{*} \mathrm{sFr} 37,50$

A. Schrauwers / B. Poolman

Synthetische Biologie - Der Mensch als Schöpfer?

In diesem reich illustrierten Band erzählen die Autoren, wie das Erbgut, die DNA, entschlüsselt wurde und wie man sie heute synthetisch produzieren kann. Dabei geht es in diesem Buch nicht um neue Fabeltiere oder rekonstruierte Mammuts, vielmehr versuchen die Autoren, dem Leser einen Einblick darüber zu geben, was zurzeit in der Synthetischen Biologie geschieht und wohin sich dieses Forschungsgebiet vermutlich entwickeln wird.

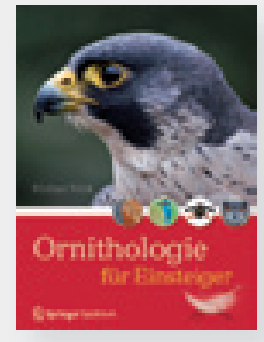

2013, 292 S. 260 Abb. in Farbe. Geb.

ISBN 978-3-8274-2324-5 $€$ (D) $39,95|€(A) 41,07|$ ${ }^{*} \mathrm{sFr} 50,00$

Michael Wink

Ornithologie für Einsteiger

Mit diesem reich illustrierten Band legt Michael Wink, Fachmann für Ornithologie, ein wissenschaftlich fundiertes, dennoch verständliches Werk über die verschiedenen Facetten der Ornithologie vor. Es dient zum einen als Einstieg in das faszinierende Hobby des Birdwatching, zum anderen liefert es dem erfahrenen Vogelbeobachter wissenschaftlich interessante Aspekte der Vogelbiologie ( Evolution und Systematik der Vögel, Anatomie und Physiologie, Ernährung, Entwicklung, Verhalten, Zug u. v. m.).

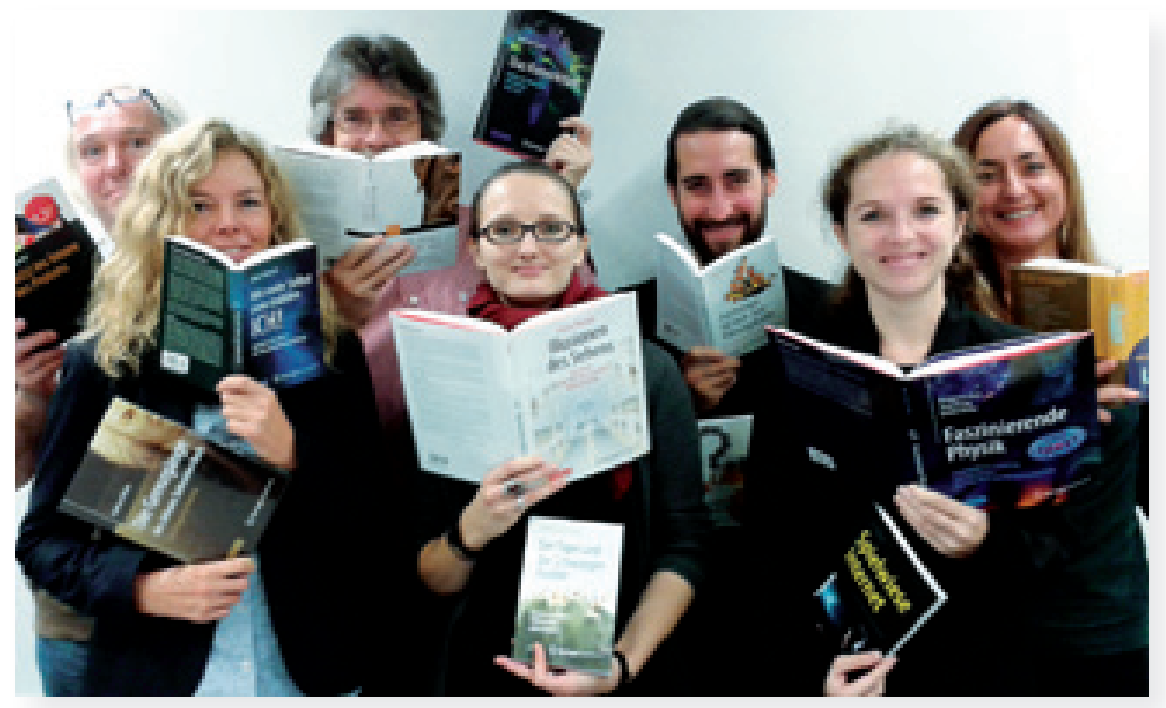

\section{Neu auf Facebook:}

\section{Springer Spektrum Sachbuchforum}

BÜCHERFANS aufgepasst! Seit kurzem gibt es eine neue Springer Spektrum-Facebookseite für alle, die gerne (Sach)Bücher lesen: SPRINGER SPEKTRUM SACHBUCHFORUM. Hier finden Fans der Seite künftig Infos (und Diskussionen) zu aktuellen Neuerscheinungen, kostenlose Lese-Exemplare, Chats mit Autoren u. v. m.

2013, XI, 470 S. 2 Abb. Brosch

Kelly G. Lambert

Lehrmeister Ratte

Was können uns ganz gewöhnliche Laborratten über das Menschsein verraten? Die Biopsychologin Kelly Lambert meint: sehr viel. Sie führt seit nunmehr fünfundzwanzig Jahren Forschungen an Ratten und anderen Nagetieren durch und ist dabei zu einer überraschenden Erkenntnis gekommen: Mit ihrer Anpassungsfähigkeit und ihren besonderen Lebensgewohnheiten vermögen uns diese bescheidenen Tierchen so einiges darüber beizubringen, wie wir als Menschen ein besseres Leben führen können. Von Arbeitsethos Betreuung des Nachwuchses und zur Erhaltung der eigenen Gesundheit kann die Laborratte für uns alle ein - zugegebenermaßen ungewöhnliches - Vorbild sein.

„Ein faszinierendes Buch, das sich dank Lamberts anregendem und witzigem Stil nur so wegliest ... Es ist auch absolut überzeugend. Tatsächlich könnten Sie sich bei dem nächsten Dilemma, vor dem Sie stehen, die überraschende Frage stellen: Was würde eine Ratte jetzt tun?" Richmond Times Dispatch 